\title{
Proposta de um Índice de Destinação de Resíduos Sólidos Industriais
}

\author{
Purpose of an Industrial Solid Waste Destination Index
}

\section{Hosmanny Mauro Goulart Coelho}

Engenheiro Civil. Mestre e Doutorando em Saneamento, Meio Ambiente e Recursos Hídricos pela Universidade Federal de Minas Gerais (UFMG). Especialista em Fontes Alternativas de Energia pela Universidade Federal de Lavras (UFLA) e MBA em Gerenciamento de Projetos pela Fundação Getúlio Vargas (FGV)

\author{
Liséte Celina Lange \\ Química e Doutora em Tecnologia Ambiental pelo Imperial College - London University. \\ Professora Associada do Departamento de Engenharia Sanitária e Ambiental da UFMG \\ Lucas Filipe Lucena Jesus \\ Graduando em Engenharia Civil pela UFMG. Pesquisador de Iniciação Científica do Programa Institucional de Bolsas de Iniciação Científica/Conselho \\ Nacional de Desenvolvimento Científico e Tecnológico (PIBIC/CNPq) pela UFMG \\ Matheus Rennó Sartori \\ Graduando em Engenharia Civil pela UFMG. Pesquisador de Iniciação Científica do PIBIC/CNPq pela UFMG
}

\begin{abstract}
Resumo
A pesquisa envolveu o desenvolvimento do Índice de Destinação de Resíduos Sólidos Industriais, composto por 7 sub-índices que abrangem 20 indicadores desenvolvidos, envolvendo os princípios de não geração, minimização, reaproveitamento, reciclagem, tratamento, estocagem e disposição final de resíduos. O marco referencial que orientou as avaliações do Índice de Destinação de Resíduos Sólidos Industriais foi obtido a partir da realização de pesquisas em relatórios ambientais e documentos auditáveis, bem como em visitas técnicas em indústrias dos setores automotivo, cimenteiro, laticínios, siderúrgico e têxtil. O Índice de Destinação de Resíduos Sólidos Industriais desenvolvido vem sendo simulado a partir de dados reais. Em simulação feita para uma indústria siderúrgica o Índice de Destinação de Resíduos Sólidos Industriais somatório obtido foi 0,645 (REGULAR) e o produtório foi 0,785 (BOM). Os resultados obtidos se mostram satisfatórios para avaliação e apoio aos gestores industriais na tomada de decisões.
\end{abstract}

Palavras-chave: resíduos sólidos industriais; gerenciamento de resíduos; índices e indicadores.

\section{Abstract}

The research involved the development of the Industrial Solid Waste Destination Index, formed by 7 sub-index that involve 20 indicators, considering the principles of non-generation, minimization, reuse, recycling, treatment, storage and disposal of waste. The reference that guided the evaluations of Industrial Solid Waste Destination Index was derived from the conduct of research in environmental reports and auditable documents, as well as visits to the industries of automotive, cement, dairy, steel and textiles. The developed Industrial Solid Waste Destination Index has been simulated with real data obtained. In the simulation carried out for the steel industry was obtained Industrial Solid Waste Destination Index summation equal to 0,645 (REGULAR) and Industrial Solid Waste Destination Index product equal to 0,785 (GOOD). The results have been satisfactory in terms of evaluation and support of industrial managers in making their decisions.

Keywords: industrial solid waste; waste management; indicators and indexes. 


\section{Introdução}

Cada vez mais, o setor produtivo em diferentes países está incorporando em seus custos aqueles relacionados à questão ambiental, implicando necessidades de mudanças significativas nos padrões de produção, comercialização e consumo. Essas mudanças respondem a normas e dispositivos legais rígidos de controle (nacionais e internacionais), associados a um novo perfil de consumidor (FIESP, 2003).

Nesse sentido, as empresas têm reconhecido, seja por iniciativa própria ou movida por forças externas, a importância da busca por alternativas para melhoria de seu desempenho ambiental.

Para a Federação das Indústrias do Estado De São Paulo (FIESP, 2003), a melhoria na relação com o meio ambiente é capaz de otimizar a produtividade dos recursos utilizados, implicando benefícios diretos para a empresa, fundamentados nos princípios da Produção Mais Limpa (P+L), em que se incluem: economias de matéria-prima e insumos, resultantes do processamento mais eficiente e da sua substituição, reutilização ou reciclagem; menor consumo de água; e redução do custo de atividades envolvidas nas descargas ou no manuseio, transporte e descarte de resíduos.

$\mathrm{O}$ adequado gerenciamento de resíduos sólidos adquire caráter especial principalmente por assimilar-se facilmente a ideia de que uma redução na geração de resíduos converte-se em economia de matéria-prima, traduzindo-se claramente na redução dos custos de operação.

\section{Gerenciamento de resíduos sólidos}

Segundo a US Environmental Protection Agency (USEPA, 1992), o gerenciamento de resíduos sólidos urbanos ou industriais contempla, obrigatoriamente, as etapas de geração, coleta, armazenamento, transporte e a destinação (tratamento ou disposição final). A destinação deve obedecer ao clássico conceito da hierarquia do gerenciamento de resíduos sólidos, que se orienta da não geração até a destinação final, nessa ordem de prioridade, passando pela minimização da geração de resíduos, reutilização, reciclagem e tratamento (neste trabalho entende-se que a minimização da geração de resíduos consiste numa forma de destinação).

Contudo, apesar de existirem esforços no sentido de avaliar conceitos como o de $\mathrm{P}+\mathrm{L}$, observa-se uma lacuna quanto a mecanismos de avaliação da destinação de resíduos sólidos, que potencialmente auxiliariam as tomadas de decisão acerca desse assunto.

\section{Índices e indicadores ambientais}

Duas das ferramentas mais utilizadas para avaliação do desempenho ambiental são os índices e os indicadores.
Segundo a European Environment Agency (EEA, 2005), um indicador é uma medida geralmente quantitativa que pode ser usada para ilustrar e comunicar fenômenos complexos de maneira simples, fornecendo uma pista sobre assuntos significativos ou tornando perceptível uma tendência ou fenômeno que não é imediatamente observável. Já os índices ambientais podem ser definidos como um conjunto de indicadores agregados por meio de uma formulação matemática, que propiciam uma visão geral de fenômenos que dependem de um grande número de variáveis

A Organização para Cooperação e Desenvolvimento Econômico (OECD) foi a primeira organização internacional a desenvolver e publicar um conjunto de indicadores ambientais, no início da década de 1990. Segundo o modelo adotado pela organização, os indicadores podiam ser classificados como sendo: i) de pressão, que refletem a causa de um fenômeno; ii) de estado, que quantificam o estado de algum elemento do meio ambiente; iii) ou de resposta, que refletem a reação a uma pressão previamente estabelecida.

Saisana e Tarantola (2002) descreveram o método mais comum de desenvolvimento de índices como constituído pelas seguintes etapas:

- decisão do fenômeno a ser estudado;

- $\quad$ seleção de indicadores, que devem se pautar na relevância, na abrangência do tema escolhido e na facilidade e qualidade dos dados necessários a sua obtenção;

- estudo das relações entre os indicadores;

- normalização e atribuição dos pesos; e teste de robustez e sensibilidade, que tem o intuito de verificar a aplicabilidade e a abrangência do índice.

Apesar desses procedimentos estarem claramente definidos, a etapa de seleção de indicadores consiste num momento crítico, dado o elevado caráter subjetivo da escolha. Um dos métodos mais utilizados para resolver problemas com elevado grau de subjetividade, amplamente utilizado no meio científico, consiste no método Delphi.

\section{O método Delphi}

O método Delphi consiste numa metodologia de pesquisa que se caracteriza pela consulta a um grupo de especialistas, com o objetivo de atingir um consenso em relação a algum problema ou assunto. Resumidamente, essa consulta é feita por meio de questionários, que são reenviados até a convergência das respostas.

De forma geral, uma pesquisa é passível de ser desenvolvida por meio do Delphi quando (LINSTONE, TUROFF, 2002): o problema não se presta a uma solução analítica precisa, mas pode ser minimizado quando julgado de forma coletiva; a heterogeneidade 
das pessoas a serem consultadas deve ser preservada, como uma forma de validar o estudo; e quando essa heterogeneidade é acentuada, gerando intensa divergência ideológica, devendo o processo de comunicação ser feito anonimamente.

A aplicação do método deve respeitar alguns princípios básicos, indo desde a troca de informações entre os painelistas, de forma a eliminar o teor de individualidade dos estudos, até o anonimato das respostas, para que a opinião de painelistas amplamente reconhecidos não influencie os demais pontos de vista. Além disso, deve ser preservada a possibilidade de revisão das respostas com base na resposta do grupo consultado, o que é conseguido pela aplicação dos questionários em várias rodadas, atingindo a convergência das respostas.

Diante desse contexto, o presente trabalho tem como objetivo desenvolver indicadores e propor um Índice de Destinação de Resíduos Sólidos Industriais (IDRSI), utilizando o método Delphi, para aplicação em cinco diferentes setores da indústria de transformação (automotivo, cimenteiro, laticínio, siderúrgico e têxtil), focando a geração e a destinação de resíduos, constituindo-se numa ferramenta robusta para auxiliar a avaliação, a prevenção e o controle da poluição industrial, e ainda embasar as tomadas de decisão.

\section{Metodologia}

A metodologia adotada na elaboração do IDRSI se baseou nas diretrizes da OECD (2008A) e pode ser resumida nas seguintes etapas posteriormente detalhadas:

- realização de uma pesquisa Delphi, com o objetivo de, a partir dela, poderem ser estabelecidos o conjunto final dos indicadores e seus respectivos pesos;

- estabelecimento dos critérios para seleção dos indicadores, com base na pesquisa Delphi, obtendo-se, assim, o conjunto final de indicadores a constituir o índice;

- definição dos pesos e normalização dos indicadores selecionados;

- definição da formulação matemática do IDRSI;

- definição de procedimento para o cálculo do IDRSI caso haja dados inexistentes ou nulos;

- definição de valores padrão de desempenho para os indicadores selecionados para cálculo do IDRSI em concordância com a formulação matemática adotada;

- calibração e simulações do cálculo do IDRSI;

- definição das limitações do IDRSI.

\section{Realização da pesquisa Delphi}

\section{Pré-seleção de indicadores}

Para pré-definir os indicadores foram consultados documentos contendo indicadores ambientais, dentre os quais se podem destacar:
- indicadores integrantes de Relatórios de Desempenho Ambiental (RADA) de indústrias;

- $\quad$ indicadores sugeridos pela norma internacional ISO 14.031 (ABNT, 2004);

- $\quad$ indicadores propostos pela OECD (2001; 2008B);

- $\quad$ indicadores propostos pelo Programa das Nações Unidas para o Meio Ambiente (PNUMA) da Organização das Nações Unidas (ONU);

- indicadores de relatórios de sustentabilidade de indústrias dos cinco setores estudados;

- indicadores preconizados pela Global Reporting Initiative (GRI, 2006).

Após extensa pesquisa na literatura mundial, realizada ao longo de 2 anos, observando as diferenças entre indicadores, analisando a aplicabilidade dos indicadores existentes e identificando lacunas e dificuldades no que tange à avaliação do gerenciamento de resíduos sólidos, foram pré-definidos 31 indicadores agrupados em 7 subíndices: minimização da geração de resíduos (IMR), de reaproveitamento (IRR), de reciclagem (IRC), de coprocessamento (ICO), de incineração (IIN), de disposição final (IDF) e de estocagem (IES).

Os indicadores propostos neste trabalho se enquadram no modelo Pressão - Estado - Resposta, desenvolvido pela OECD (2001; 2008B) para indicadores ambientais e internacionalmente aceitos. Dentre os 31 indicadores pré-selecionados para compor o IDRSI, 7 apresentam características de indicadores de Resposta e os outros 24 de Pressão.

\section{Pré-teste e seleção dos painelistas}

A partir dos indicadores pré-selecionados foi realizado um pré-teste envolvendo dez painelistas. Em virtude da unanimidade dos resultados, todos os 31 indicadores foram mantidos para serem avaliados na pesquisa de opinião.

Para a aplicação da primeira rodada da pesquisa de opinião, foi criteriosamente definida uma amostra de 330 especialistas de todas as regiões do Brasil, divididos em 3 grupos alvos: 90 acadêmicos da área de resíduos sólidos; 90 técnicos de órgãos ambientais estaduais brasileiros; 150 profissionais do setor de resíduos sólidos. A Tabela 1 apresenta um resumo dos painelistas, em função do grupo alvo e da região brasileira.

\section{Elaboração do formulário}

O formulário para preenchimento dos painelistas foi elaborado para que os especialistas enumerassem escores para cada um dos 31 indicadores passíveis de compor o IDRSI, de acordo com a seguinte escala:

5. muito importante;

4. importante;

3. desejável;

2. não relevante;

1. dispensável. 
Além disso, foi solicitado aos painelistas que indicassem os cinco indicadores que julgassem mais importantes dentre os demais. Após a elaboração do formulário de preenchimento, foram enviados e-mails personalizados a cada um dos 330 painelistas contendo, em cada um deles, uma carta de apresentação da pesquisa proposta e um formulário de preenchimento.

Na primeira rodada da pesquisa, os formulários foram enviados entre 6 e 10 de março de 2010, admitindo-se como prazo limite para recebimento dos formulários preenchidos a data de 11 de abril. Após o recebimento das respostas, foram extraídas a média, a mediana, a moda e a frequência acumulada das modas dos escores atribuídos pelos painelistas antes do envio dos formulários referentes à segunda rodada.

$\mathrm{Na}$ segunda rodada, foi enviado o mesmo formulário de preenchimento, porém com a adição das informações estatísticas supracitadas para cada indicador. Dessa vez, os formulários foram enviados no dia 2 de maio, sendo o dia 16 de maio a data limite de recebimento. A partir dessa data, os dados obtidos na segunda etapa foram tabulados e, no dia 20 de maio de 2010, foram enviados e-mails aos painelistas informando sobre o resultado final da pesquisa.

\section{Critérios de seleção dos indicadores}

Os resultados da pesquisa Delphi foram utilizados para serem estabelecidos critérios para seleção dos indicadores que efetivamente viriam compor o IDRSI, bem como para atribuir pesos a cada deles, de forma similar ao trabalho realizado por Ribeiro (2005). Foram eliminados os indicadores que: obtiveram, no geral, médias ou modas inferior às notas 4 e 5 ; não foram selecionados pelos três grupos alvos simultaneamente; ou apresentaram frequência acumulada da moda menor que 50\%

\section{Definição dos pesos e normalização dos indicadores selecionados}

O peso de cada indicador selecionado para compor o IDRSI foi definido a partir das frequências acumuladas da moda das notas

Tabela 1 - Resumos dos painelistas dos grupos alvos da pesquisa de opinião por região brasileira

\begin{tabular}{|c|c|c|c|c|}
\hline \multirow{2}{*}{$\begin{array}{l}\text { Região } \\
\text { brasileira }\end{array}$} & \multicolumn{3}{|c|}{ Grupos alvos da pesquisa } & \multirow{2}{*}{$\begin{array}{c}\text { Total de } \\
\text { painelistas }\end{array}$} \\
\hline & Acadêmicos & $\begin{array}{l}\text { Técnicos de } \\
\text { órgãos ambientais }\end{array}$ & $\begin{array}{l}\text { Profissionais } \\
\text { do setor }\end{array}$ & \\
\hline Centro-Oeste & 4 & 7 & 10 & 21 \\
\hline Nordeste & 14 & 8 & 4 & 26 \\
\hline Norte & 5 & 5 & 2 & 12 \\
\hline Sudeste & 54 & 61 & 127 & 242 \\
\hline Sul & 13 & 9 & 7 & 29 \\
\hline TOTAL & 90 & 90 & 150 & 330 \\
\hline
\end{tabular}

do indicador obtidas por meio dos resultados da pesquisa Delphi. As frequências foram ponderadas em função da quantidade de indicadores em um mesmo subíndice.

A etapa de normalização dos indicadores teve o objetivo de fazer com que todos eles atingissem valores na faixa de 0 a 1 , tanto mais próximos da unidade quanto melhor for a destinação, conforme estabelecido pela hierarquia do gerenciamento de resíduos sólidos. Para tanto foram utilizadas equações com logaritmo de base 10, similar ao aplicado na normalização do Índice de Desenvolvimento Humano (IDH).

\section{Definição da formulação matemática do IDRSI}

A formulação matemática do índice tem a função de agregar os indicadores nos subíndices já definidos e combinar esses últimos para atingir o valor de cálculo final do IDRSI. Foram utilizadas as formulações de somatório e produtório, mostradas nas Equações 1 e 2 .

$I D R S I=\sum_{i=1}^{n} w i q i$

Equação 1

$I D R S I=\prod_{i=1}^{n} q_{i}{ }^{w i}$ Equação 2

onde:

wi: peso atribuído a cada indicador, cujo somatório é igual a 1; qi: valor normalizado do indicador $\mathrm{i}$;

i: cada um dos indicadores de destinação de resíduos sólidos incluído no índice;

n: número total de indicadores do índice.

A partir do cálculo IDRSI, cujo resultado pode variar entre 0 e 1, pode-se classificar determinada indústria quanto ao desempenho em relação à destinação de seus resíduos, nas faixas de variação apresentadas na Tabela 2

Sendo assim, pode-se avaliar as destinações de resíduos sólidos de indústrias de diferentes setores industriais, tendo-se como base o valor do IDRSI, o que pode auxiliar na tomada de decisão dos gestores de resíduos dessas empresas.

\section{Definição de valores padrão de desempenho do IDRSI}

Durante o período de setembro de 2008 a dezembro de 2009, foram visitadas 27 indústrias de diversos setores do Estado de Minas Gerais com o intuito de conhecer a realidade do gerenciamento de resíduos sólidos industriais.

Durante as visitas foram colhidas informações, a partir de um formulário previamente elaborado para tal fim, sobre os locais de geração de resíduos, sua coleta, acondicionamento, transporte e 
destinação. Os principais tópicos abordados pela pesquisa foram: quantidade total de resíduos gerados; quantidade de resíduos classes I e II gerados; formas de destinação dos resíduos gerados; e iniciativas tomadas visando à minimização ou a não geração de resíduos.

Além dessas visitas técnicas, foi realizada uma extensa pesquisa nos documentos públicos da Fundação Estadual do Meio Ambiente (Feam) com o objetivo de conhecer melhor os procedimentos adotados pelas empresas no que tange ao gerenciamento e à destinação dos resíduos sólidos industriais, e observar a disponibilidade e a forma de divulgação dos dados.

Foram analisados também os Inventários de Resíduos Sólidos Industriais elaborados pela Feam e publicados nos anos de 2003, 2009 e 2010, bem como o Caderno de Produção Mais Limpa, também elaborado pela Feam e publicando em 2009, em que é apresentado o desenvolvimento do Índice de Produção Mais Limpa para a indústria de transformação do Estado de Minas Gerais.

Com as informações colhidas nessas atividades, foram selecionados cinco setores da indústria de transformação a serem abordados neste trabalho, a saber: automotivo, cimenteiro, laticínios, siderúrgico e têxtil. A abrangência desse número de setores industriais por parte do IDRSI tornou necessária uma coleta de dados de indicadores de empresas pertencentes a cada um deles com a finalidade executar as simulações para avaliação do índice proposto.

Contudo, a execução de tais simulações requereu que fossem estabelecidos os valores mínimos e máximos de referência de cada um dos indicadores componentes do IDRSI para cada uma dos cinco setores industriais. Estes valores - benchmarks - foram pesquisados tanto na literatura específica quanto em relatórios de sustentabilidade de indústrias de todo o mundo. Deve-se observar que a principal vantagem em utilizar benchmarks consiste na possibilidade contínua de adequação do indicador aos avanços nas tecnologias de produção e destinação de resíduos.

\section{Definição do tratamento de dados inexistentes}

Com o intuito de superar parcialmente a inexistência de dados, foi adotado o critério de adotar valores nulos nos casos em que os dados reais não estão disponíveis. Deve ser ressaltado, porém, que quanto mais bem consolidado for o levantamento dos dados para iniciar o cálculo do IDRSI, mais realistas são as conclusões a que o índice pode conduzir.

\section{Calibração e simulações do cálculo do IDRSI}

As simulações realizadas para validação do IDRSI basearam-se primeiramente em dados secundários de empresas dos cinco setores industriais em estudo, sendo seguidas da calibração do modelo em função dos resultados obtidos. Em seguida, essa formatação do índice proposto foi testada adotando dados primários. Como exemplo da aplicação do IDRSI são apresentados neste trabalho os resultados de uma simulação realizada para uma indústria do setor siderúrgico.

\section{Resultados e Discussão}

\section{Resultado da pesquisa de opinião - método Delphi}

A Tabela 3 apresenta o número de respostas para cada grupo alvo. Dos 330 painelistas iniciais, 27 foram descartados, pois os emails retornaram com erro, restado 307 e-mails válidos, dos quais 171 permaneceram respondendo até o final da pesquisa. Nota-se que os painelistas do setor acadêmico apresentaram a maior taxa de adesão, provavelmente, devido ao reconhecimento da importância de participação em pesquisas de opinião, para verificar a convergência a respeito de determinado tema. Destaca-se também que cerca de $70 \%$ dos painelistas são da Região Sudeste, devido à concentração de profissionais da área atuando nessa região.

A pesquisa Delphi teve uma excelente participação com taxa de adesão de 55,7\%; acredita-se que isto se deu em função da qualidade do formulário, no que diz respeito à facilidade e à rapidez para seu preenchimento. Além disso, o número de participantes se mostra superior quando comparado a outras pesquisas (Tabela 4).

Portanto, acredita-se que a pesquisa Delphi realizada logrou êxito e que os resultados podem ser considerados satisfatórios para a continuação deste trabalho. Diante disso, após concluir a recepção das respostas da pesquisa de opinião, os resultados foram

Tabela 2 - Faixas de classificação do Índice de Destinação de Resíduos Sólidos Industriais

\begin{tabular}{lc|} 
Faixas de Variação & Classificação \\
\hline 0,9 a 1,0 & Excelente \\
0,8 a 0,9 & Muito bom \\
0,7 a 0,8 & Bom \\
0,6 a 0,7 & Regular \\
0,5 a 0,6 & Tolerável \\
0,4 a 0,5 & Ruim \\
$<0,4$ & Muito ruim
\end{tabular}

Tabela 3 - Indicadores definidos para serem utilizados no índice de Destinação de Resíduos Sólidos Industriais

\begin{tabular}{|lccc} 
Grupo alvo & $\begin{array}{c}\text { E-mails } \\
\text { válidos }\end{array}$ & $\begin{array}{c}\text { Respostas } \\
\text { recebidas }\end{array}$ & $\begin{array}{c}\text { Taxa de } \\
\text { adesão (\%) }\end{array}$ \\
\hline Acadêmicos & 86 & 52 & 60,5 \\
\hline Profissionais do setor & 141 & 78 & 55,3 \\
\hline Técnicos de órgãos ambientais & 80 & 41 & 51,3 \\
\hline TOTAL & 307 & 171 & 55,7
\end{tabular}


Tabela 4 - Comparação entre os resultados de pesquisas utilizando 0 método Delphi

\begin{tabular}{lccc} 
Pesquisa & $\begin{array}{c}\text { Número de } \\
\text { especialistas }\end{array}$ & $\begin{array}{c}\text { Número de } \\
\text { respostas } \\
\text { recebidas }\end{array}$ & $\begin{array}{c}\text { Taxa de } \\
\text { Adesão } \\
(\%)\end{array}$ \\
\hline $\begin{array}{l}\text { Fatores intervenientes no } \\
\text { desempenho de unidades de } \\
\text { triagem e compostagem de }\end{array}$ & 43 & 19 & 44,2 \\
$\begin{array}{l}\text { RSU (VIMIEIRO, 2009) } \\
\begin{array}{l}\text { Índice de qualidade para água } \\
\text { bruta (SOUZA, LIBÂNIO, 2009) }\end{array}\end{array}$ & 24 & 18 & 75,0 \\
$\begin{array}{l}\text { Indicadores de desenvolvimento } \\
\text { sustentável para indústria têxtil } \\
\text { (PADILHA, 2005) }\end{array}$ & 81 & 45 & 56,0 \\
\hline
\end{tabular}

RSU: resíduos sólidos urbanos.

tabulados e tratados estatisticamente, de forma a possibilitar a definição dos indicadores e respectivos pesos a serem utilizados na composição do IDRSI, conforme apresentados a seguir.

\section{Composição do IDRSI}

A partir dos resultados do Delphi e dos critérios definidos relatados anteriormente, foram eliminados 11 dos 31 indicadores pré-selecionados. Logo, restaram 20 indicadores para compor o IDRSI conforme apresentado na Tabela 5.

\section{Pesos obtidos para os indicadores do IDRSI}

Os pesos obtidos para cada um dos indicadores são apresentados na Tabela 6, onde se observa que o somente o subíndice de minimização de resíduos (IMR) representa quase 20\% do IDRSI demonstrando a preocupação dos painelistas com a não geração e a diminuição dos resíduos sólidos. Além disso, ao se considerar a soma dos subíndices de reaproveitamento (IRR), reciclagem (IRC) e do IMR, o percentual atinge 53,8\% do IDRSI, evidenciando ainda mais a importância dessas atividades para a sustentabilidade ambiental do setor industrial. Além disso, ressalta-se a importância de se analisar os percentuais de cada subíndice, pois essa avaliação possibilita ao gestor industrial visualizar em que etapa do gerenciamento de resíduos sólidos da indústria ele deve atuar para melhorar o seu IDRSI.

A Tabela 6 mostra que os subíndices ICO, IIN, IDF e IES representam $46,2 \%$ do IDRSI.

\section{Definição das limitações do IDRSI}

Com o intuito de evitar a obtenção de resultados e interpretações equivocadas, foram considerados os seguintes limites de aplicação do IDRSI:

- os indicadores foram desenvolvidos para cinco setores da indústria de transformação, não aplicam, portanto, à indústria extrativa;
- os indicadores desenvolvidos consideraram como alternativas de tratamento apenas a incineração, o coprocessamento e o aterramento, por serem as mais utilizadas no Brasil.

\section{Simulações do IDRSI}

Como exemplos da aplicação do IDRSI, apresentam-se os resultados obtidos em uma simulação realizada para uma indústria do setor siderúrgico. A Tabela 7 apresenta os dados de entrada utilizados no cálculo do IDRSI. Para efetuar os cálculos do IDRSI, os dados foram inseridos pelo pesquisador responsável pelo trabalho, utilizando-se as relações da Tabela 5.

Analisando a Tabela 7 constata-se um decréscimo da produção e da geração de resíduos no ano de 2008 em relação a 2007, provavelmente ocorrido em função da crise econômica mundial iniciada naquele ano. Observa-se ainda que os percentuais de resíduos reaproveitados e reciclados somados alcançaram percentuais muito elevados, 98,0 e 96,5\% nos anos de 2007 e 2008, respectivamente, destacando a preocupação da indústria com a minimização e, consequentemente, com a sustentabilidade ambiental de seu processo produtivo.

As Tabelas 8 e 9 apresentam os resultados obtidos para o cálculo do IDRSI para uma indústria do setor siderúrgico pelo método somatório e produtório, respectivamente. Salienta-se que para o cálculo do IDRSI produtório foram desconsiderados os valores dos indicadores iguais a 0 .

Na Tabela 8, constata-se que a quantidade de resíduos aterrados prejudicou o subíndice IDF.

Pelas Tabelas 8 e 9, observa-se que o valor do IDRSI calculado pela equação do somatório foi de 0,645 (REGULAR) inferior ao obtido pelo do produtório que foi de 0,785 (BOM). Isso se deve, provavelmente, a uma maior sensibilidade do produtório para melhorias no sistema de gerenciamento. De fato, deve-se salientar que a equação do produtório é mais sensível à existência de valores extremos (mínimo e máximo) nos subíndices, podendo mascarar os resultados do IDRSI e conduzir a conclusões precipitadas e, muitas vezes, inadequadas. Além disso, analisando-se a Tabela 9, verifica-se que o cálculo do IDRSI produtório somente foi possível porque os valores nulos de alguns indicadores não foram considerados na multiplicação.

Ainda observando-se as Tabelas 8 e 9, verifica-se que os valores dos subíndices IMR, IRR e IRC influenciaram mais fortemente no IDRSI, obtido pela equação do produtório do que no somatório. Entretanto, o cálculo do índice por ambas as equações é importante para possibilitar comparações e análises, de forma a auxiliar o gestor industrial na identificação de qual valor do IDRSI é mais realista frente à situação de seu gerenciamento de resíduos, com o intuito de promover melhorias internas. Salienta-se que, em geral, o método somatório é uma opção mais vantajosa quando se pretende conhecer o rendimento geral da empresa em todos os quesitos analisados. 
Finalmente, destaca-se que os dados de entrada (Tabela 7) foram previamente calculados para serem introduzidos nas Tabelas 8 e 9 como valores normalizados e, efetivamente, serem utilizados nos cálculos do IDRSI.

\section{Conclusões}

Na realização do presente trabalho, o método Delphi mostrouse uma importante e interessante ferramenta para a pesquisa de opinião e para busca de consenso entre especialistas de uma área, fazendo uso da experiência e do conhecimento acumulado por esses na obtenção de respostas sobre um determinado assunto. A pesquisa Delphi teve uma excelente participação com taxa de adesão de $55,7 \%$, provavelmente obtida em função da qualidade do formulário, no que tange a facilidade e rapidez para o seu preenchimento
Deve-se salientar que uma das grandes dificuldades que se encontrou foi a obtenção de dados do setor industrial para estabelecer o benchmarking (valores de referência) de forma a permitir comparações mais realistas entre as indústrias analisadas.

A simulação da indústria do setor siderúrgico revelou que o valor do IDRSI obtido pelo método somatório igual a 0,645 (REGULAR) foi inferior ao IDRSI obtido pelo método produtório que foi de 0,785 (BOM), mas precisamente obteve-se uma diferença de 0,140 no valor do índice, que corresponde a uma variação positiva de aproximadamente $22 \%$.

Por fim, destaca-se que os indicadores e o IDRSI desenvolvido atenderam aos objetivos propostos, uma vez que vêm sendo utilizados para simulações de cenários reais e seus resultados demonstram aderência à realidade dos setores industriais investigados na pesquisa.

Tabela 5 - Indicadores definidos para serem utilizados no índice Índice de Destinação de Resíduos Sólidos Industriais

\begin{tabular}{|c|c|}
\hline Código & Descrição do indicador \\
\hline MR-1 & $\begin{array}{l}\text { [total de resíduos gerados no ano atual }(\mathrm{t}) / \text { total de produtos produzidos no ano atual }(\mathrm{t})] / \\
\text { [total resíduos gerados no ano anterior }(\mathrm{t}) / \text { total produtos produzidos no ano anterior }(\mathrm{t})]\end{array}$ \\
\hline MR-2 & $\begin{array}{l}\text { [resíduos classe I (perigosos) gerados no ano atual }(\mathrm{t}) / \text { total produtos produzidos no ano atual }(\mathrm{t})] \text { / } \\
\text { [resíduos classe I (perigosos) gerados no ano anterior }(\mathrm{t}) / \text { total produtos produzidos no ano anterior }(\mathrm{t})]\end{array}$ \\
\hline MR-4 & $\begin{array}{l}\text { [resíduos classe I (perigosos) gerados no ano atual }(\mathrm{t}) / \text { total resíduos gerados no ano atual }(\mathrm{t})] \text { / } \\
\text { [resíduos classe I (perigosos) gerados no ano anterior }(\mathrm{t}) / \text { total resíduos gerados no ano anterior }(\mathrm{t})]\end{array}$ \\
\hline RR-1 & percentual total de resíduos reutilizados ou reaproveitados em relação ao total de resíduos gerados no ano atual (\%) \\
\hline RR-2 & $\begin{array}{l}\text { [total de resíduos reaproveitados no ano atual }(\mathrm{t}) / \text { total de resíduos gerados no ano atual }(\mathrm{t})] \text { / } \\
\text { [total resíduos reaproveitados no ano anterior }(\mathrm{t}) / \text { total de resíduos gerados no ano anterior }(\mathrm{t})]\end{array}$ \\
\hline RR-4 & $\begin{array}{l}\text { percentual de substituição de combustível não renovável na produção decorrente da reutilização ou reaproveitamento de resíduos no ano } \\
(\%)\end{array}$ \\
\hline RR-5 & percentual de substituição de matéria-prima decorrente da reutilização ou reaproveitamento de resíduos no ano (\%) \\
\hline RR-6 & percentual de resíduo total transferido a outra indústria para substituição de matéria-prima ou aproveitamento energético (\%) \\
\hline $\mathrm{RC}-1$ & percentual de total de resíduos reciclados em relação ao total de resíduos gerados no ano atual (\%) \\
\hline $\mathrm{RC}-2$ & $\begin{array}{l}\text { [total de resíduos reciclados no ano atual }(\mathrm{t}) / \text { total de resíduos gerados no ano atual }(\mathrm{t})] \text { / } \\
\text { [total resíduos reciclados no ano anterior }(\mathrm{t}) / \text { total de resíduos gerados no ano anterior }(\mathrm{t})]\end{array}$ \\
\hline $\mathrm{RC}-4$ & percentual de substituição de combustível não renovável na produção decorrente da reciclagem de resíduos no ano (\%) \\
\hline $\mathrm{RC}-5$ & percentual de substituição de matéria-prima decorrente da reciclagem de resíduos no ano atual (\%) \\
\hline $\mathrm{CO}-3$ & $\begin{array}{l}\text { [total de resíduos coprocessados no ano atual }(\mathrm{t}) / \text { total de resíduos gerados no ano atual (t)] / } \\
\text { [total resíduos coprocessados no ano anterior }(\mathrm{t}) / \text { total de resíduos gerados no ano anterior }(\mathrm{t})]\end{array}$ \\
\hline $\mathrm{CO}-4$ & $\begin{array}{l}\text { [resíduos classe I coprocessados no ano atual (t)/resíduos classe I gerados no ano atual (t)] / } \\
\text { [resíduos classe I coprocessados no ano anterior (t)/resíduos classe I gerados no ano anterior (t)] }\end{array}$ \\
\hline IN-3 & $\begin{array}{l}\text { [total de resíduos incinerados no ano atual }(\mathrm{t}) / \text { total de resíduos gerados no ano atual }(\mathrm{t})] \text { / } \\
\text { [total resíduos incinerados no ano anterior }(\mathrm{t}) / \text { total de resíduos gerados no ano anterior }(\mathrm{t})]\end{array}$ \\
\hline IN-4 & $\begin{array}{l}\text { [resíduos classe I incinerados no ano atual (t)/resíduos classe I gerados no ano atual (t)] / } \\
\text { [resíduos classe I incinerados no ano anterior (t)/resíduos classe I gerados no ano anterior (t)] }\end{array}$ \\
\hline DF-3 & $\begin{array}{l}\text { [total de resíduos aterrados no ano atual (t)/total de resíduos gerados no ano atual (t)] / } \\
\text { total resíduos aterrados no ano anterior (t)/total de resíduos gerados no ano anterior (t)] }\end{array}$ \\
\hline $\mathrm{DF}-4$ & $\begin{array}{l}\text { [resíduos classe I aterrados no ano atual (t)/resíduos classe I gerados no ano atual (t)] / } \\
\text { [resíduos classe I aterrados no ano anterior (t)/resíduos classe I gerados no ano anterior (t)] }\end{array}$ \\
\hline ES-3 & $\begin{array}{l}\text { [total de resíduos estocados no ano atual (t)/total de resíduos gerados no ano atual (t)] / } \\
\text { [total resíduos estocados no ano anterior (t)/total de resíduos gerados no ano anterior (t)] }\end{array}$ \\
\hline ES-4 & $\begin{array}{l}\text { [resíduos classe I estocados no ano atual (t)/resíduos classe I gerados no ano atual (t)] / } \\
\text { [resíduos classe I estocados no ano anterior (t)/resíduos classe I gerados no ano anterior (t)] }\end{array}$ \\
\hline
\end{tabular}


Tabela 6 - Pesos utilizados obtidos para os Indicadores do Índice de Destinação de Resíduos Sólidos Industriais

\begin{tabular}{|c|c|c|c|}
\hline Subíndice & Código do indicador & Pesos obtidos & Percentual do IDRSI (\%) \\
\hline \multirow[t]{3}{*}{ Minimização (IMR) } & MR-1 & 0,068 & \multirow[t]{3}{*}{19,6} \\
\hline & MR-2 & 0,066 & \\
\hline & MR-4 & 0,062 & \\
\hline \multirow[t]{5}{*}{ Reaproveitamento (IRR) } & RR-1 & 0,038 & \multirow[t]{5}{*}{17,3} \\
\hline & RR-2 & 0,035 & \\
\hline & RR-4 & 0,033 & \\
\hline & RR-5 & 0,033 & \\
\hline & RR-6 & 0,034 & \\
\hline \multirow[t]{4}{*}{ Reciclagem (IRC) } & $\mathrm{RC}-1$ & 0,046 & \multirow[t]{4}{*}{16,9} \\
\hline & $\mathrm{RC}-2$ & 0,042 & \\
\hline & $\mathrm{RC}-4$ & 0,039 & \\
\hline & $\mathrm{RC}-5$ & 0,042 & \\
\hline \multirow[t]{2}{*}{ Coprocessamento (ICO) } & $\mathrm{CO}-3$ & 0,058 & \multirow[t]{2}{*}{11,8} \\
\hline & $\mathrm{CO}-4$ & 0,060 & \\
\hline \multirow[t]{2}{*}{ Incineração (IIN) } & IN-3 & 0,059 & \multirow[t]{2}{*}{11,7} \\
\hline & IN-4 & 0,058 & \\
\hline \multirow[t]{2}{*}{ Disposição final (IDF) } & DF-3 & 0,062 & \multirow[t]{2}{*}{12,3} \\
\hline & DF-4 & 0,061 & \\
\hline \multirow[t]{2}{*}{ Estocagem (IES) } & ES-3 & 0,052 & \multirow[t]{2}{*}{10,4} \\
\hline & ES-4 & 0,052 & \\
\hline
\end{tabular}

IDRSI: Índice de Destinação de Resíduos Sólidos Industriais.

Tabela 7 - Dados de entrada utilizados no cálculo do Índice de Destinação de Resíduos Sólidos Industriais em indústria siderúrgica (FEAM, 2009)

\begin{tabular}{|c|c|c|}
\hline \multirow{2}{*}{ Setor industrial: siderúrgico } & \multicolumn{2}{|c|}{ Ano } \\
\hline & 2007 & 2008 \\
\hline Produção (t/ano) & 4.062 .750 & 3.287 .750 \\
\hline Quantidade total de resíduo sólido gerada (t/ano) & 758.088 & 539.672 \\
\hline Quantidade de resíduo sólido perigoso gerada (t/ano) & 81 & 3.080 \\
\hline Quantidade de resíduo sólido não perigoso gerada (t/ano) & 758.007 & 536.592 \\
\hline Quantidade total de resíduo sólido reutilizada ou reaproveitada (t/ano) & 371.878 & 260.304 \\
\hline Percentual de combustível não renovável poupado pelo reaproveitamento de resíduo (\%) & 0 & 0 \\
\hline Percentual de matéria-prima poupada devido ao reaproveitamento de resíduo (\%) & 0 & 0 \\
\hline Percentual de resíduo transferido à outra indústria para servir como matéria-prima ou combustível (\%) & 0 & 0 \\
\hline Quantidade de resíduo sólido reciclada (t/ano) & 371.878 & 260.304 \\
\hline Percentual de combustível não renovável poupado devido à reciclagem de resíduo (\%) & 0 & 0 \\
\hline Percentual de matéria-prima poupada devido à reciclagem de resíduo sólido (\%) & 0 & 0 \\
\hline Quantidade de resíduo sólido coprocessada (t/ano) & 49 & 0 \\
\hline Quantidade de resíduo sólido perigoso coprocessada (t/ano) & 49 & 0 \\
\hline Quantidade de resíduo sólido incinerada (t/ano): & 2 & 0 \\
\hline Quantidade de resíduo sólido perigoso incinerada (t/ano) & 2 & 0 \\
\hline Quantidade de resíduo sólido aterrada (t/ano) & 14.284 & 19.064 \\
\hline Quantidade de resíduo sólido perigoso aterrada (t/ano) & 30 & 3.080 \\
\hline Quantidade de resíduo sólido estocada (t/ano) & 0 & 0 \\
\hline Quantidade de resíduo sólido perigoso estocada (t/ano) & 0 & 0 \\
\hline
\end{tabular}

t/ano: tonelada ao ano. 
Tabela 8 - Resultados do cálculo do Índice de Destinação de Resíduos Sólidos Industriais (método somatório) para indústria do setor siderúrgico

\begin{tabular}{|c|c|c|c|c|c|c|c|c|c|}
\hline \multirow{2}{*}{ Subíndice } & \multirow{2}{*}{ Indicador } & \multicolumn{2}{|c|}{ Benchmarking } & \multirow{2}{*}{$\begin{array}{c}\text { Valor } \\
\text { normalizado } \\
\text { (Vnorm) }\end{array}$} & \multirow{2}{*}{$\begin{array}{l}\text { Peso do } \\
\text { indicador }\end{array}$} & \multirow{2}{*}{$\begin{array}{c}\% \\
\text { subíndice }\end{array}$} & \multirow{2}{*}{$\begin{array}{l}\text { Indicador } \\
\text { normalizado } \\
\text { x peso }\end{array}$} & \multirow{2}{*}{$\begin{array}{c}\text { Total } \\
\text { subíndice }\end{array}$} & \multirow{2}{*}{ Valor IDRSI } \\
\hline & & (Vmín) & (Vmáx) & & & & & & \\
\hline \multirow{3}{*}{ IMR } & MR-1 & 0,00308 & 0,67081 & 0,87 & 0,068 & \multirow{3}{*}{19,6} & 0,059 & \multirow{3}{*}{0,172} & \multirow{17}{*}{0,645} \\
\hline & MR-2 & 0,00038 & 0,20104 & 0,87 & 0,066 & & 0,057 & & \\
\hline & MR-4 & 0,00428 & 0,62696 & 0,90 & 0,062 & & 0,056 & & \\
\hline \multirow{4}{*}{ IRR } & RR-1 & 0,02370 & 0,80981 & 0,95 & 0,038 & \multirow{4}{*}{17,3} & 0,036 & \multirow{4}{*}{0,071} & \\
\hline & RR-4 & 0,00000 & 1,00000 & 0,00 & 0,033 & & 0,000 & & \\
\hline & RR-5 & 0,00000 & 1,00000 & 0,00 & 0,033 & & 0,000 & & \\
\hline & RR-6 & 0,00000 & 1,00000 & 0,50 & 0,034 & & 0,017 & & \\
\hline \multirow{2}{*}{ IRC } & $\mathrm{RC}-1$ & 0,04578 & 0,84117 & 0,95 & 0,046 & \multirow{2}{*}{16,9} & 0,044 & \multirow{2}{*}{0,086} & \\
\hline & $\mathrm{RC}-2$ & 0,00278 & 0,34208 & 0,50 & 0,042 & & 0,021 & & \\
\hline $\mathrm{ICO}$ & $\mathrm{CO}-4$ & 0,00000 & 1,00000 & 0,40 & 0,060 & 11,8 & 0,024 & 0,081 & \\
\hline \multirow{2}{*}{ IIN } & IN-3 & 0,00000 & 1,00000 & 0,90 & 0,059 & \multirow{2}{*}{11,7} & 0,053 & \multirow{2}{*}{0,102} & \\
\hline & IN-4 & 0,00000 & 1,00000 & 0,85 & 0,058 & & 0,049 & & \\
\hline \multirow{2}{*}{ IDF } & DF-3 & 0,00361 & 0,98731 & $-0,04$ & 0,062 & \multirow{2}{*}{12,3} & $-0,002$ & \multirow{2}{*}{0,029} & \\
\hline & DF-4 & 0,03432 & 0,98731 & 0,51 & 0,061 & & 0,031 & & \\
\hline \multirow{3}{*}{ IES } & ES-3 & 0,00000 & 1,00000 & 1,00 & 0,052 & \multirow{2}{*}{10,4} & 0,052 & \multirow{2}{*}{0,104} & \\
\hline & ES-4 & 0,00000 & 1,00000 & 1,00 & 0,052 & & 0,052 & & \\
\hline & & & & TOTAL & 1,0 & 100 & 0,645 & 0,645 & \\
\hline
\end{tabular}

Vmín: valor mínimo de referência; Vmáx: valor máximo de referência; Vnorm: valor do indicador normalizado.

Tabela 9 - Resultados do cálculo do IDRSI (método produtório) para indústria do setor siderúrgico

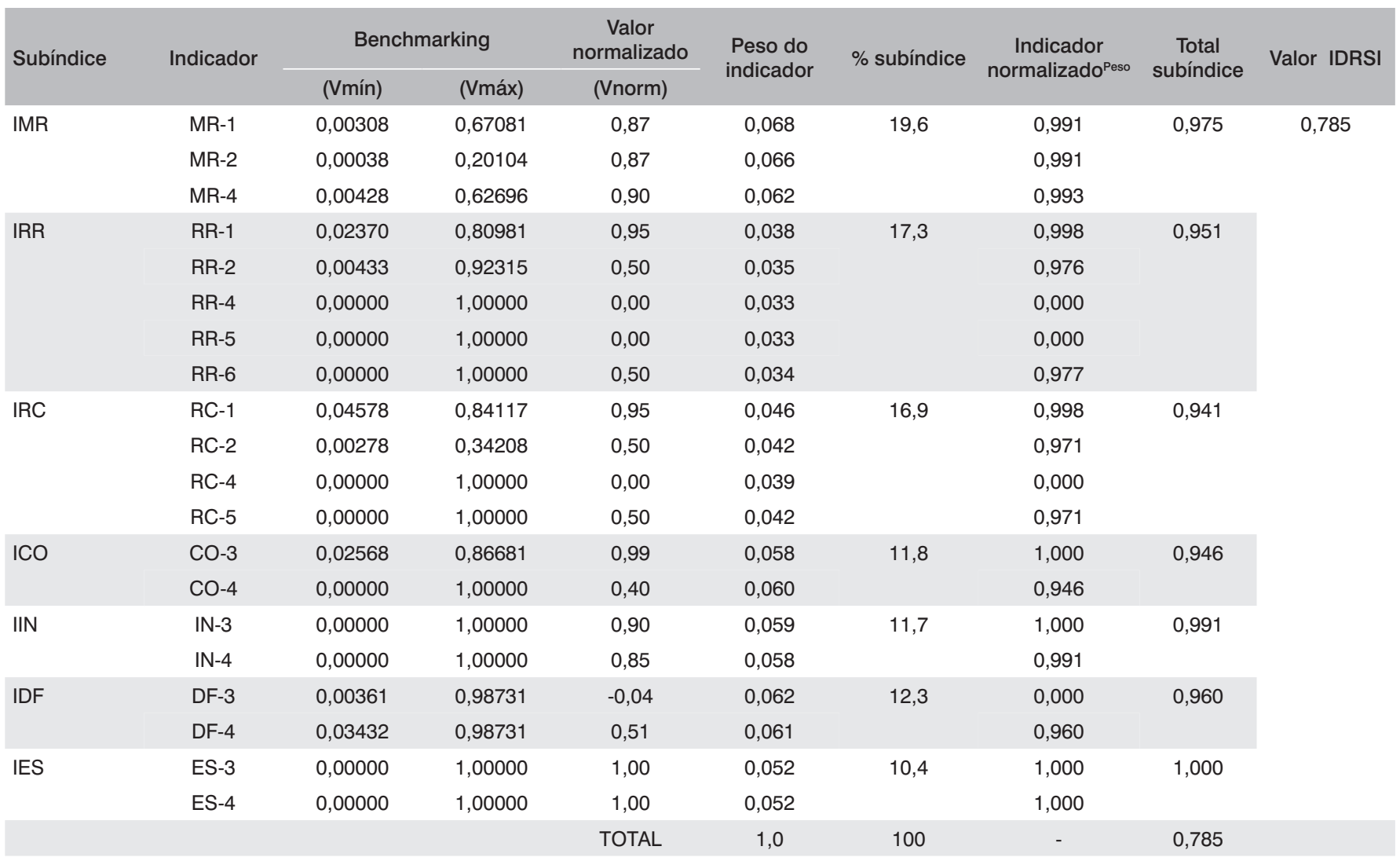

Vmín: valor mínimo de referência; Vmáx: valor máximo de referência; Vnorm: valor do indicador normalizado. 


\section{Referências}

ASSOCIAÇÃO BRASILEIRA DE NORMAS TÉCNICAS (ABNT). NBR ISO 14031 Gestão Ambiental - Avaliação de Desempenho Ambiental. Rio de Janeiro: ABNT, 2004

EUROPEAN ENVIRONMENT AGENCY (EEA). EEA core set of indicators Guide. EEA Technical report n. 1/2005. ISSN 1725-2237, 2005.

FUNDAÇÃO ESTADUAL DO MEIO AMBIENTE (Feam). Índice de produção mais limpa para indústria de transformação do Estado Minas Gerais. Belo Horizonte: Feam, 2009.

FEDERAÇÃO DAS INDÚSTRIAS DO ESTADO DE SÃO PAULO (Fiesp). Indicadores de desempenho ambiental da indústria. São Paulo: Fiesp/ Ciesp, 2003

GLOBAL REPORTING INICIATIVE (GRI). Diretrizes para relatório de sustentabilidade. Versão 3.0 (Português). São Paulo: GRI, 2006.

LINDSTONE, H.A.; TUROFF, M [Internet]. The De/phi Method: techniques and applications. EBook. 2002 [cited 2008 Ago. 19]. Available from: http://is.njit.edu/pubs/delphibook/>

ORGANIZATION FOR ECONOMIC CO-OPERATION AND DEVELOPMENT (OECD) Handbook on constructing composite indicators. Methodology and user guide: OECD, 2008A.

ORGANIZATION FOR ECONOMIC CO-OPERATION AND DEVELOPMENT (OECD). Key environmental indicators. Paris: OECD, 2008B.
ORGANIZATIONFORECONOMICCO-OPERATIONANDDEVELOPMENT (OECD). Key environmental indicators. Paris: OECD, 2001.

PADILHA, M.L.M.L. Indicadores de desenvolvimento sustentável para o setor têxtil. Tese [Doutorado]. 311p. São Paulo: Faculdade de Saúde Pública da Universidade de São Paulo, 2009.

RIBEIRO, J. C. J. Desenvolvimento de modelo para avaliação de desempenho de política pública de meio ambiente. Estudo de caso: Estado de Minas Gerais. Tese [Doutorado]. 335p. Minas Gerais: Escola de Engenharia da UFMG, 2005

SAISANA, M.; TARANTOLA, S. State-of-the-art report on current methodologies and practices for composite indicator development Joint Research Centre. Italy: European Commission, 2002.

SOUZA, M.E.T.A.; LIBÂNIO, M. Proposta de índice de qualidade para água bruta afluente a estações convencionais de tratamento. Engenharia Sanitária e Ambiental, Rio de Janeiro, v. 14, n. 4, p. 471-478, 2009.

US ENVIRONMENTAL PROTECTION AGENCY (Usepa) Decisionmakers guide to solid waste management. N. ${ }^{\circ}$ 530-R-95-023. 2. ed. Office of Solid Waste and Emergency Response, 1992.

VIMIEIRO, G.V.; LANGE, L.C. Utilização do método Delphi para definição dos fatores intervenientes no desempenho de unidades de triagem e compostagem de resíduos sólidos urbanos. In: ANAIS $25^{\circ}$ CONGRESSO DA ASSOCIAÇÃO BRASILEIRA DE ENGENHARIA SANITÁRIA E AMBIENTAL (ABES), 2009, Recife-PE, v. 1, p. 1-8, 2009. 